\title{
Inhibition characteristics of circulating current in parallel inverter driving system of mine hoist
}

\author{
Kuanfang $\mathrm{He}^{1,2} \cdot$ Yong $\mathrm{Wang}^{2} \cdot$ Dongming Xiao $^{2}$
}

Received: 17 May 2015/Revised: 25 August 2015/Accepted: 10 October 2015/Published online: 24 February 2016

(C) The Author(s) 2016. This article is published with open access at Springerlink.com

\begin{abstract}
This research investigated the outputting circulation current inhibition characteristic which are controlled by the instantaneous feedback voltage in inverter parallel driving of the mine hoist. We established a transfer function of the parallel inverters controlled by the close-loop adjustment of instantaneous voltage feedback. The influence of the parameters of the close-loop feedback circuit to the inhibition effects to the outputting circulation current is observed. After analyzing the circulating current inhibition characteristics, the proportion integration (PI) controller is introduced into the close-loop adjustment by instantaneous voltage feedback. The characteristics equation is gained to determine the PI parameters by drawing the Bode plots. The inhibition effects of the proposed controller are examined by the established simulation model of parallel inverter system. The harmonic distortion rate at the outputting voltage frequency value of 4 , $10,20,41$ and $50 \mathrm{~Hz}$, are all lower than $2.32 \%$ by the instantaneous outputting voltage feedback.
\end{abstract}

Keywords Mine hoist $\cdot$ Parallel inverters $\cdot$ Circulating current $\cdot$ Inhibition characteristic $\cdot$ Close-loop adjustment

\section{Introduction}

Parallel inverter driving system of mine hoist differs from traditional driving inverter system, which contains a parallel connection system of two independent inverter units to drive the motor. Parallel inverter driving system of mine hoist can effectively increase power levels and improve the reliability of the inverter driving system. The total current of parallel inverter driving system is equal to the sum of the two inverter units in parallel structure. If one of the inverter units goes wrong, the mine hoist system can be driven by the other one, which guarantees the continuity and safety of

Kuanfang $\mathrm{He}$

hkf91113@163.com

$1 \quad$ Engineering Research Center of Advanced Mining Equipment, Ministry of Education, Hunan University of Science and Technology, Xiangtan 411201, China

2 Provincial Key Laboratory of Mechanical Equipment Healthy Maintenance, Hunan University of Science and Technology, Xiangtan 411201, China the equipment in working condition. However, when the two inverter units in parallel structure have differences in amplitude, frequency and phase of the outputting voltage, there will induce large circulating current among the two inverters. A huge harmonic circulating current will be induced especially in the condition of the difference of dead times existed in the two inverters, which not only affects the amplitude and phase of the outputting voltage, but also causes single harmonic. The harmonic circulating current has bad influences on the electrical system, consuming large amounts of energy, causing waveform distortion of the output voltage and inducing electromagnetic interference (Hyosung and Ji 2004). Therefore, it is necessary and significant to research the inhibition technology of the harmonic circulating current in inverter parallel driving systems of mine hoist.

There are available literatures and referenced results about the inhibition technology of harmonic circulation current in parallel inverter driving system. The direct current (DC) component generated in the inverter parallel system makes the output filter inductor of inverter be saturation, uneven thermal and electrical stress of each 
electrical module, which reduce the reliability of the inverter parallel driving system (Chen et al. 2003). In order to inhibit the DC circulating current, there are some developed methods such as voltage and current double close-loop control, three-state hysteresis control, filter circuit elimination and regulation of the reference sine wave DC component (Chen et al. 2004; He and Xu 2012; He and Zhang 2008). Zhang et al. analyzed the case of two inverters sharing a rectifier module, which will produce a zero sequence circulation current through the DC bus and consume a lot of energy, reduce the efficiency of the system and cause saturation of filter reactor (2006a). Therefore, inverter units in parallel were suggested to adopt a separate rectifier modules. Zhang et al. (2006b) and Liu et al. (2011) analyzed the presence of harmonic circulating current caused by dead zone dispersion of switch, delay of driving circuit and over modulation, of which the dead zone of switch is the most influenced component. In recent years, some inhibition technologies of harmonic circulation current are proposed, including control method of the instantaneous voltage feedback, repetitive control method and dual-loop control method of voltage and current (Ogasawara et al. 1992; Kawakami et al. 1994).

Considering the dead zone of switch being the most influenced component to the presence of harmonic circulating current in parallel inverter driving system, regarding the error of the output voltage caused by the dispersion of the dead zone as a harmonic disturbance, an inhibition method of harmonic circulating current controlled by the instantaneous feedback voltage is proposed. The outputting circulation current inhibition characteristic is also investigated.

\section{Inhibition principle by the instantaneous voltage feedback}

Figure 1 shows the regulation and control frame diagram of inhibition principle by the instantaneous outputting voltage feedback. $K_{\mathrm{V}}$ is the coefficient of voltage feedback.

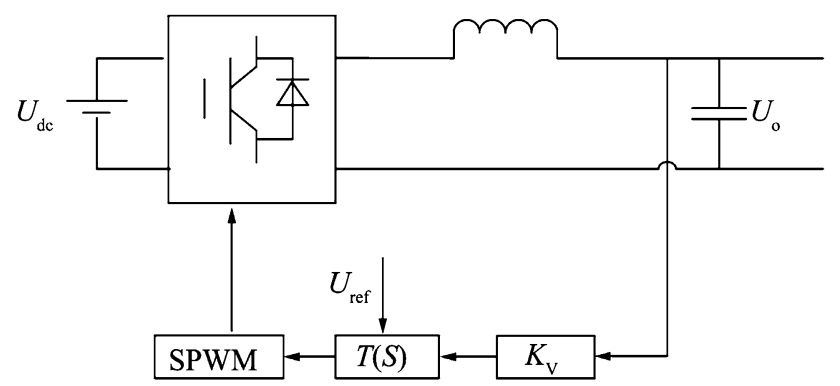

Fig. 1 The regulation and control frame diagram of inhibition principle of the instantaneous outputting voltage feedback
The error of voltage is obtained by detecting the instantaneous voltage across the capacitor by comparing to the reference sine voltage signal $U_{\text {ref }}(s)$. The error of voltage generates a modulation wave by the regulator $T(S)$. After conducting a comparison of modulation wave to the carrier one, the SPWM wave is obtained. The SPWM wave is the driving signal to the inverter.

A transfer function of the inhibition principle by the instantaneous outputting voltage feedback is established based on Fig. 1, and is shown in Fig. 2. In the model, $U_{\text {ref }}$ is the standard reference voltage, $T(s)$ is regulator of the instantaneous voltage feedback, $K_{\mathrm{PWM}}$ is an equivalent gain for PWM, $K_{\mathrm{v}}$ is the coefficient of voltage feedback, $r$ is the equivalent circuit resistance, $L$ is the filter inductor, $C$ is the filter capacitor, and $U_{\mathrm{o}}(s)$ is the outputting voltage.

The transfer function of the closed-loop operation can be obtained:

$$
\begin{aligned}
U_{\mathrm{o}}(\mathrm{s})= & \frac{K_{\mathrm{PWM}} T(s)}{L C s^{2}+r C s+K_{\mathrm{PWM}} K_{\mathrm{v}} T(s)+1} U_{\mathrm{ref}}(s) \\
& -\frac{1}{L C s^{2}+r C s+K_{\mathrm{PWM}} K_{\mathrm{v}} T(s)+1} U_{\mathrm{e}}(s) \\
& -\frac{L s+r}{L C s^{2}+r C s+K_{\mathrm{PWM}} K_{\mathrm{v}} T(s)+1} I_{\mathrm{o}}(s)
\end{aligned}
$$

According to the Eq. (1), the outputting voltage feedback regulator increases attenuation gain of the error of voltage caused by dead zone of switching component of the circuit, and has a good inhibiting effect on harmonic circulating current. Reasonable adjustment of $T(s)$ parameters can improve the stability of the system and the quality of the outputting waveform. At the same time, the circuit parameters of $L, C, r, K_{\mathrm{V}}, K_{\mathrm{PWM}}$ are appropriately selected to impose an impact on the inhibition of harmonic circulating current.

Let $B_{e}(\mathrm{~s})=L C s^{2}+r C s+K_{\mathrm{PWM}} K_{\mathrm{v}} T(\mathrm{~s})+1$, then the Eq. (1) can be written as:

$U_{\mathrm{o}}(\mathrm{s})=\frac{K_{\mathrm{PWM}} T(s)}{B_{\mathrm{e}}(s)} U_{\text {ref }}(s)-\frac{1}{B_{\mathrm{e}}(s)} U_{\mathrm{e}}(s)-\frac{L s+r}{B_{\mathrm{e}}(s)} I_{\mathrm{o}}(s)$

The harmonic circulating current Eq. (3) can be obtained by Eq. (2):

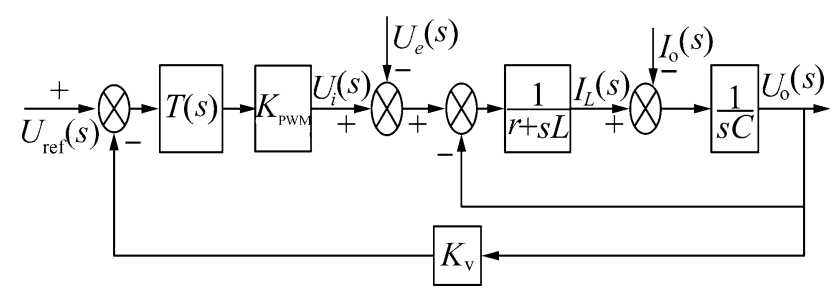

Fig. 2 The function model of the inhibition principle of the instantaneous outputting voltage feedback 


$$
\begin{aligned}
I_{\mathrm{H}}(s)= & \frac{K_{\mathrm{PWM}} T(s)}{L s+r+Z B_{\mathrm{e}}(s)} \frac{U_{\mathrm{ref} 1}(s)-U_{\mathrm{ref} 2}(s)}{2} \\
& +\frac{1}{L s+r+Z B_{\mathrm{e}}(s)} \frac{U_{\mathrm{e} 2}(s)-U_{\mathrm{e} 1}(s)}{2}
\end{aligned}
$$

where $H(s)=\frac{1}{L s+r+Z B_{\mathrm{e}}(s)}$, it can be seen that the circulation error is mainly inhibited by $H(s)$, the harmonic circulating current can be reduced by decreasing the gain of $H(s)$ and increasing the $B_{\mathrm{e}}(s)$. According to Eq. (1), the increasing of $B_{\mathrm{e}}(s)$ reduce the outputting harmonic voltage. Meanwhile, according to $H(s)$, inhibition effect is greater when the $Z, L$ and $r$ are larger. However, the voltage drop and the distortion of the outputting waveform will be greater when the parameters of $Z, L$ and $r$ are selected by too much large values, and the stability of the system will be affected by too large value of $B_{\mathrm{e}}(s)$. Thus, appropriate values should be selected for $Z, L, r$ and $B_{\mathrm{e}}(s)$. According to the expression of $B_{\mathrm{e}}(s)$, it is mainly controlled by $T(s)$.

\section{Determination of parameters of the instantaneous voltage feedback}

$T(\mathrm{~s})$ is adjusted by adopting PI regulator that is a simple and effective method to be easily implemented. The expression of the $T(\mathrm{~s})$ can be written as:

$T(s)=K_{P}+K_{i} \frac{1}{s}$

Substituting Eq. (4) into Eq. (1), the characteristic equation can be obtained as:

$B_{\mathrm{e}}(\mathrm{s})=L C \mathrm{~s}^{3}+r C s^{2}+\left(K_{\mathrm{PWM}} K_{\mathrm{v}} K_{\mathrm{P}}+1\right) s+K_{\mathrm{PWM}} K_{\mathrm{v}} K_{i}$

It can be seen from Eq. (5) that the characteristic equation has three roots. The dominant roots are $s_{1,2}=-\zeta \omega_{n} \pm j \omega_{n} \sqrt{1-\zeta^{2}}$, where $\zeta$ is the damping ratio, $\omega_{n}$ is the natural frequency. The no dominant root $s_{3}=-n \zeta \omega_{n}$, and $n$ is the normal number with the value ranging from five to ten. The characteristic equation of the system can be written:

$$
\begin{aligned}
B_{e}(s) & =\left(s-s_{1}\right) \cdot\left(s-s_{2}\right) \cdot\left(s-s_{3}\right) \\
& =\left(s^{2}+2 \zeta \omega_{n} s+\omega_{n}^{2}\right)\left(s+n \zeta \omega_{n}\right)
\end{aligned}
$$

According to the pole assignment method, the following expression can be obtained:

$\left\{\begin{array}{l}K_{\mathrm{P}}=\frac{\left(2 n \zeta^{2}+1\right) \omega_{n}^{2} L C-1}{K_{\mathrm{PWM}} K_{\mathrm{V}}} \\ K_{i}=\frac{n \zeta \omega_{n}^{3} L C}{K_{\mathrm{PWM}} K_{\mathrm{V}}}\end{array}\right.$

Equation (7) shows the PI parameters of $T(\mathrm{~s})$ controller. It can be seen from Eq. (7) that $K_{\mathrm{PWM}}, K_{\mathrm{V}}, L, C$ are parameters of the inverter unit circuit, $\zeta, \omega_{n}, n$ are the parameters to be determined. The parameters of inverter unit circuit are assumed as follows: $L=1 \mathrm{mH}, C=30 \mu \mathrm{F}$, $r=0.1 \Omega, K_{\mathrm{PWM}}=33.3$ and $K_{\mathrm{V}}=0.0257$. Substitution of Eq. (7) into the regulator $T(s)$, the closed-loop transfer function of system can be written as:

$$
\begin{aligned}
U_{\mathrm{o}}(s)= & \frac{K_{\mathrm{PWM}} K_{\mathrm{P}} s+K_{\mathrm{PWM}} K_{i}}{L C \mathrm{~s}^{3}+r C s^{2}+\left(K_{\mathrm{PWM}} K_{\mathrm{v}} K_{\mathrm{P}}+1\right) s+K_{\mathrm{PWM}} K_{\mathrm{v}} K_{i}} U_{\mathrm{ref}}(s) \\
& -\frac{s}{L C \mathrm{~s}^{3}+r C s^{2}+\left(K_{\mathrm{PWM}} K_{\mathrm{v}} K_{\mathrm{P}}+1\right) s+K_{\mathrm{PWM}} K_{\mathrm{v}} K_{i}} U_{\mathrm{e}}(s) \\
& -\frac{L s^{2}+r s}{L C \mathrm{~s}^{3}+r C s^{2}+\left(K_{\mathrm{PWM}} K_{\mathrm{v}} K_{\mathrm{P}}+1\right) s+K_{\mathrm{PWM}} K_{\mathrm{v}} K_{i}} I_{\mathrm{o}}(s)
\end{aligned}
$$

Let $\left\{\begin{array}{l}G_{1}=\frac{K_{\mathrm{PWM}} K_{\mathrm{P}} s+K_{\mathrm{PWM}} K_{i}}{L C \mathrm{~s}^{3}+r C s^{2}+\left(K_{\mathrm{PWM}} K_{\mathrm{v}} K_{\mathrm{P}}+1\right) s+K_{\mathrm{PWM}} K_{\mathrm{v}} K_{i}} \\ G_{2}=\frac{s}{L C \mathrm{~s}^{3}+r C s^{2}+\left(K_{\mathrm{PWM}} K_{\mathrm{v}} K_{\mathrm{P}}+1\right) s+K_{\mathrm{PWM}} K_{\mathrm{v}} K_{i}} \\ Z=\frac{L s^{2}+r s}{L C \mathrm{~s}^{3}+r C s^{2}+\left(K_{\mathrm{PWM}} K_{\mathrm{v}} K_{\mathrm{P}}+1\right) s+K_{\mathrm{PWM}} K_{\mathrm{v}} K_{i}}\end{array}\right.$

The Bode plots are drawn by Eq. (9), the system performance influenced by different $\zeta, \omega_{n}, n$ are analyzed. Table 1 shows the PI coefficients of the $T(s)$ regulator by different values of $n$.

The Bode plot depending on the different values of $n$ is shown in Fig. 3. According to the Bode plot of $G_{1}$, the logarithmic amplitude-frequency characteristic substantially coincides with the horizontal axis in low-frequency range. The gain of a given reference voltage of $U_{\text {ref }}\left(j \omega_{0}\right)$ is one when $\left|G_{1}\left(\omega_{0}\right)\right| \approx 1, \omega_{0}$ is the system operating frequency, the outputting voltage $U_{\mathrm{o}}\left(j \omega_{0}\right)$ can track the given reference voltage $U_{\text {ref }}\left(j \omega_{0}\right)$. In the Fig. 3, the arrow means the increasing direction of $\mathrm{n}$. The bandwidth of $G_{1}$ and the moderate amplitude become wider as the increasing of $n$. According to the Bode plots of $G_{2}$ and $Z$, the gain is

Table 1 The coefficient of $T(s)$ regulator under different $n$

\begin{tabular}{lcccccc}
\hline$n$ & 4 & 6 & 8 & 10 & 12 & 20 \\
\hline$K_{\mathrm{P}}$ & 0.979 & 1.837 & 2.696 & 3.554 & 4.413 & 7.848 \\
$K_{i}$ & 12763.21 & 19144.81 & 25526.42 & 31908.00 & 38289.62 & 63816.00 \\
\hline
\end{tabular}


(a)

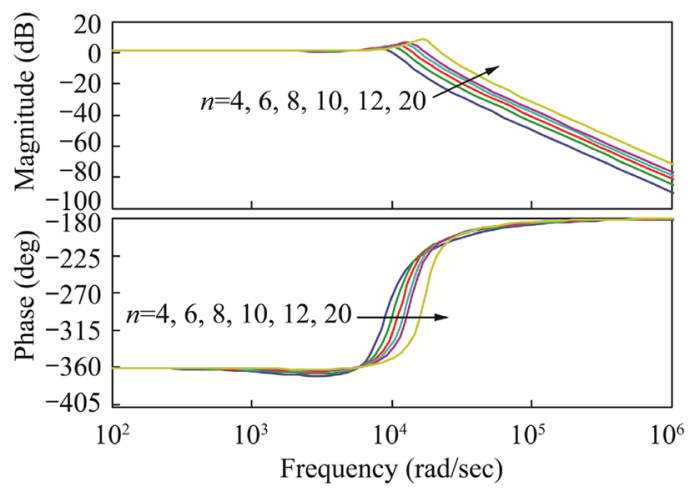

(b)

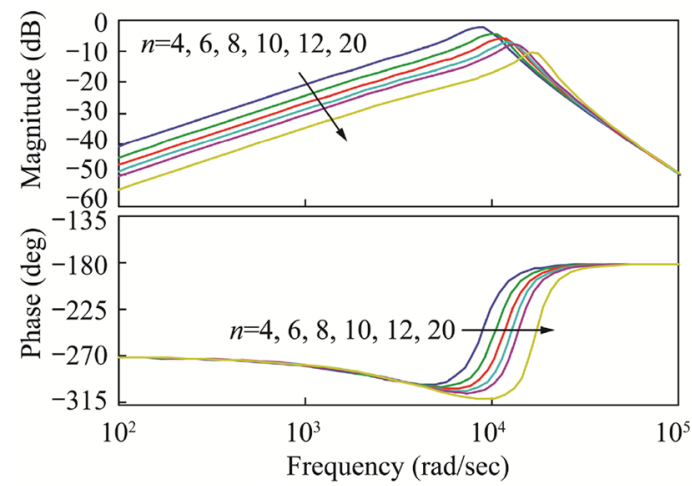

(c)

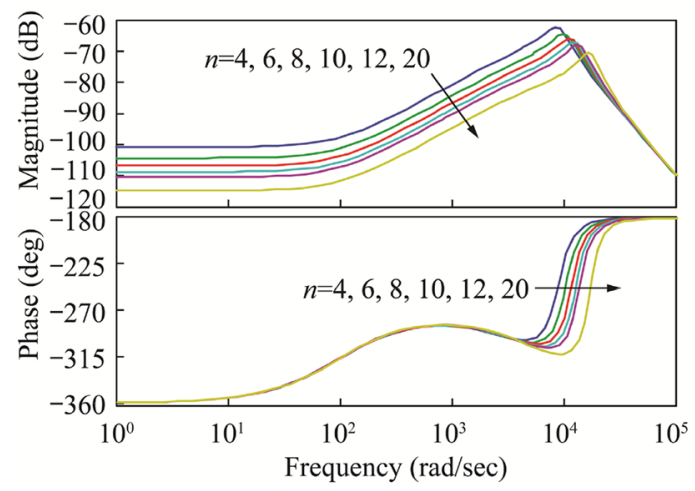

Fig. 3 The impact on the system performance under different $n$. a $G_{1}$ Bode diagram. b $G_{2}$ Bode diagram. c $Z$ Bode diagram

relatively small in middle and low frequency ranges, which have a damping effect on the error voltage $U_{\mathrm{e}}(s)$ caused by the dead zone and voltage drop of the circuit. The gain of $G_{2}$ and $Z$ decreases as the increasing of $n$. (a)

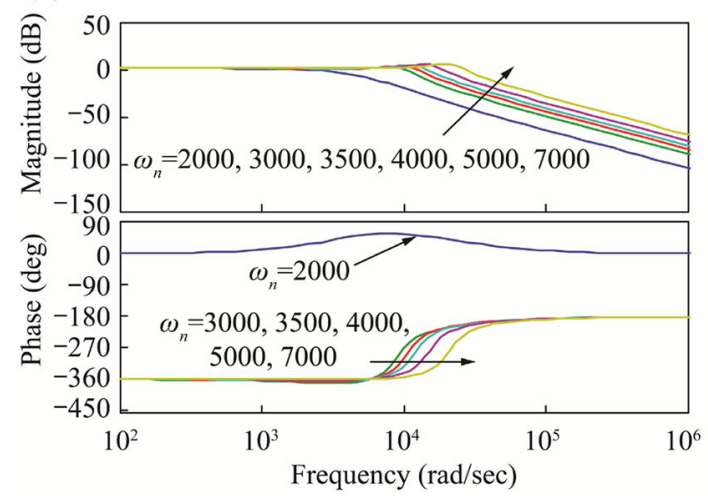

(b)

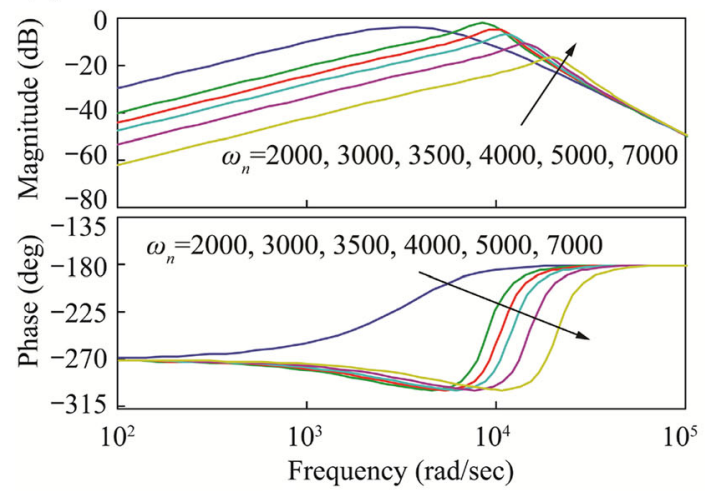

(c)

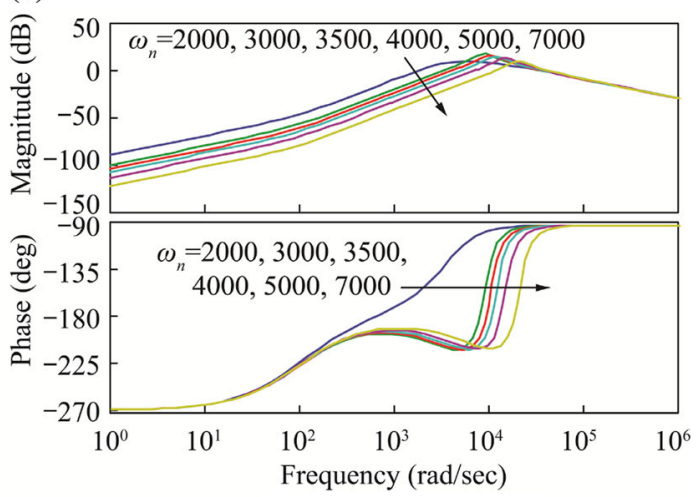

Fig. 4 The effect to the system performance under different $\omega_{n}$. a $G_{1}$ Bode diagram. b $G_{2}$ Bode diagram. c $Z$ Bode diagram

Table 2 shows the PI coefficients of the $T(s)$ regulator by different values of $\omega_{n}$.

The Bode plot depending on the different values of $n$ are drawn in Fig. 4. According to the Bode plot of $G_{1}$, the

Table 2 The coefficients of $T(s)$ regulator under different $\omega_{n}$

\begin{tabular}{llcccrr}
\hline$\omega_{n}$ & 2000 & 3000 & 3500 & 4000 & 5000 & 7000 \\
\hline$K_{P}$ & -0.187 & 1.041 & 1.837 & 2.757 & 4.965 & 10.853 \\
$K_{i}$ & 3572.00 & 12055.50 & 19144.81 & 28576.00 & 55812.51 & 153149.5 \\
\hline
\end{tabular}


Table 3 The coefficients of $T(s)$ regulator under different $\zeta$

\begin{tabular}{lccccrr}
\hline$\zeta$ & 0.4 & 0.6 & 0.707 & 0.8 & 1.2 & 1.8 \\
\hline$K_{\mathrm{P}}$ & 0.085 & 1.120 & 1.837 & 2.558 & 6.680 & 15.95 \\
$K_{i}$ & 10831.58 & 16247.37 & 19144.81 & 21663.16 & 32494.74 & 48742.11 \\
\hline
\end{tabular}
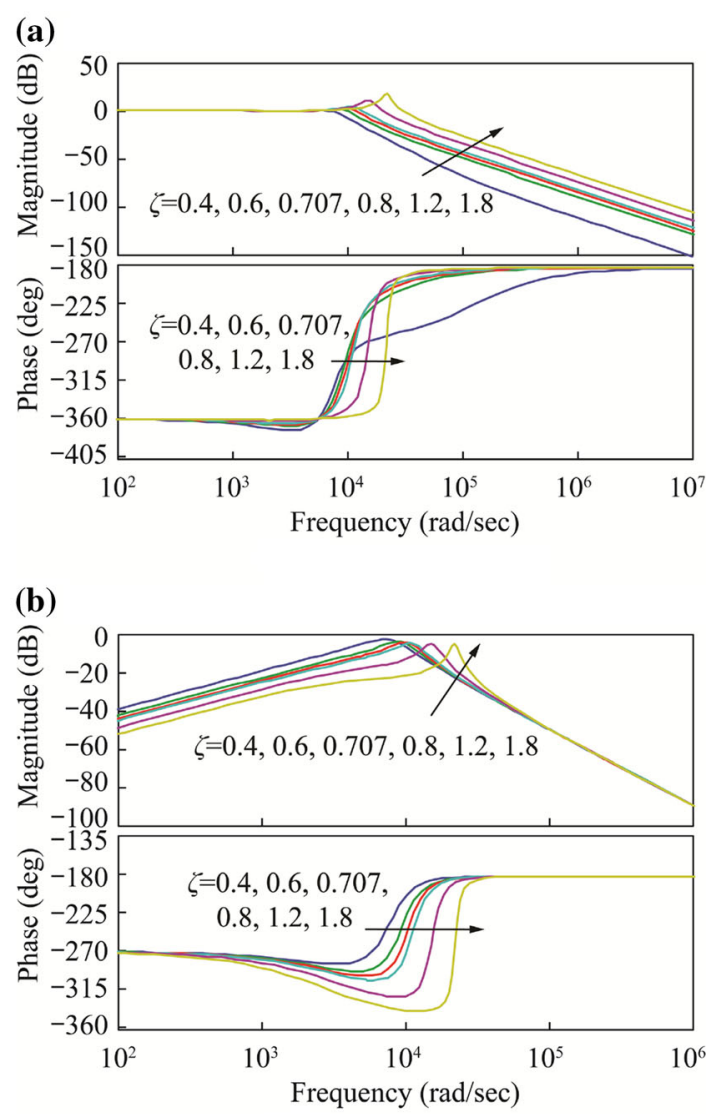

(c)

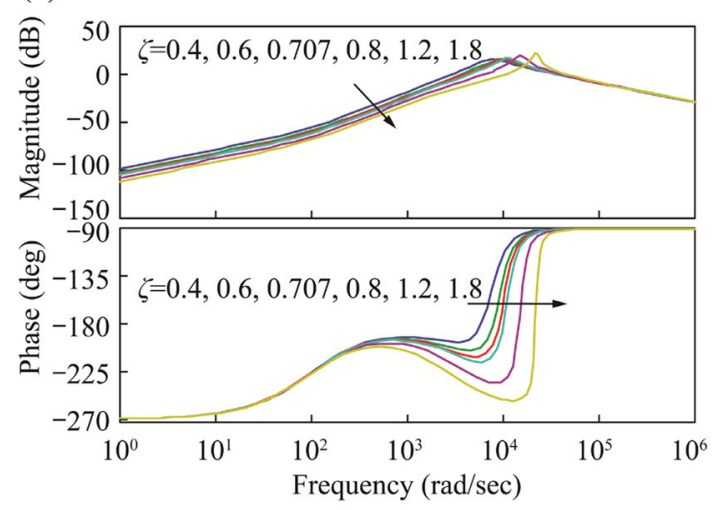

Fig. 5 The effect to the system performance under different $\zeta$. a $G_{1}$ Bode diagram. b $G_{2}$ Bode diagram. c $Z$ Bode diagram system is of the least stabilization when $\omega_{n}=2000$. The bandwidth of $G_{1}$ becomes wider than that of $n$ in middle and low frequency ranges as the increasing of $\omega_{n}$. Therefore, $\omega_{n}$ has more impact on steady-state error of the system.

According to the Bode plots of $G_{2}$ and $Z$, the gains of $G_{2}$ and $Z$ are negative, which inhibit the voltage error $U_{\mathrm{e}}(s)$ caused by the dead zone and voltage drop of the circuit. The gain of $G_{2}$ and $Z$ become smaller with more effect of inhibition as increasing of $\omega_{n}$.

Table 3 shows the PI coefficients of the $T(s)$ regulator by different values of the damping ratio $\zeta$. The Bode plots depending on the different values of $\zeta$ are shown in Fig. 5.

According to the Bode plot of $G_{1}$, when $\zeta \geq 1.2$, the gains are positive near the corner frequency, the ability to recurrence signal decreases, and the noise will be amplified at high frequency. Therefore, the most appropriate range of $\zeta$ is $0.5-1.0$. Therefore, let $\zeta=0.707$.

In summary, the ability to track the given standard voltage becomes enlarged as the increasing of $\omega_{n}$, decreased as the increase of $n$ and $\zeta$. The changing of $\zeta$ has more impact on the system compared to the changing of $n$. The ability to inhibit the harmonic voltage caused by the dead zone and the voltage drop of the circuit are enhanced as increasing of $\omega_{n}, n$ and $\zeta$. Considering above analysis, the parameter values are determined by $\omega_{n}=3500, n=6$ and $\zeta=0.707$, which are substituted into Eq. (7), the PI coefficients of $T(s)$ regulator are determined as follows:

$\left\{\begin{array}{l}K_{P}=1.837 \\ K_{i}=19144.81\end{array}\right.$

\section{Numerical simulation and results}

Figure 6 is an parallel inverter driving simulation model established by MATLAB/Simulink. The motor is driven by two parallel inverter units after the electric power pass through the RLC filter. The parameters in the model are set as follows: DC voltage $V_{1}=V_{2}=514 \mathrm{~V}$, for RLC filter, $R=80 \mathrm{e}^{-3} \Omega, L=3 \mathrm{e}^{-3} \Omega, C=1.2 \mathrm{e}^{-3} \mathrm{~F}$, induction motor with parameters of are $5.5 \mathrm{~kW}, 400 \mathrm{~V}, 1460 \mathrm{rpm}, 50 \mathrm{~Hz}$, $20 \mathrm{~nm}$ and RMS of $50 \mathrm{~Hz}$. 


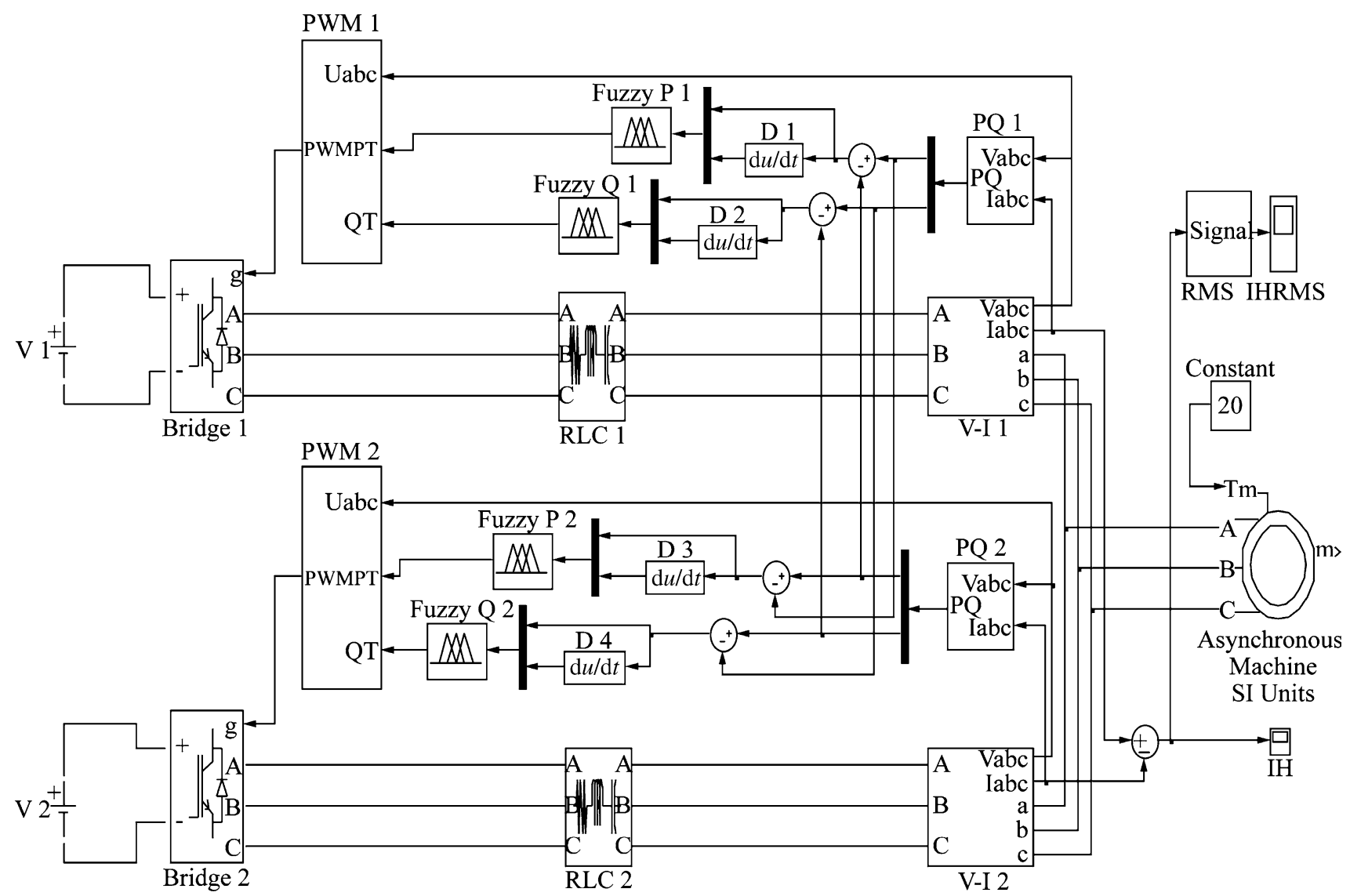

Fig. 6 The simulation model of two frequency inverters in parallel

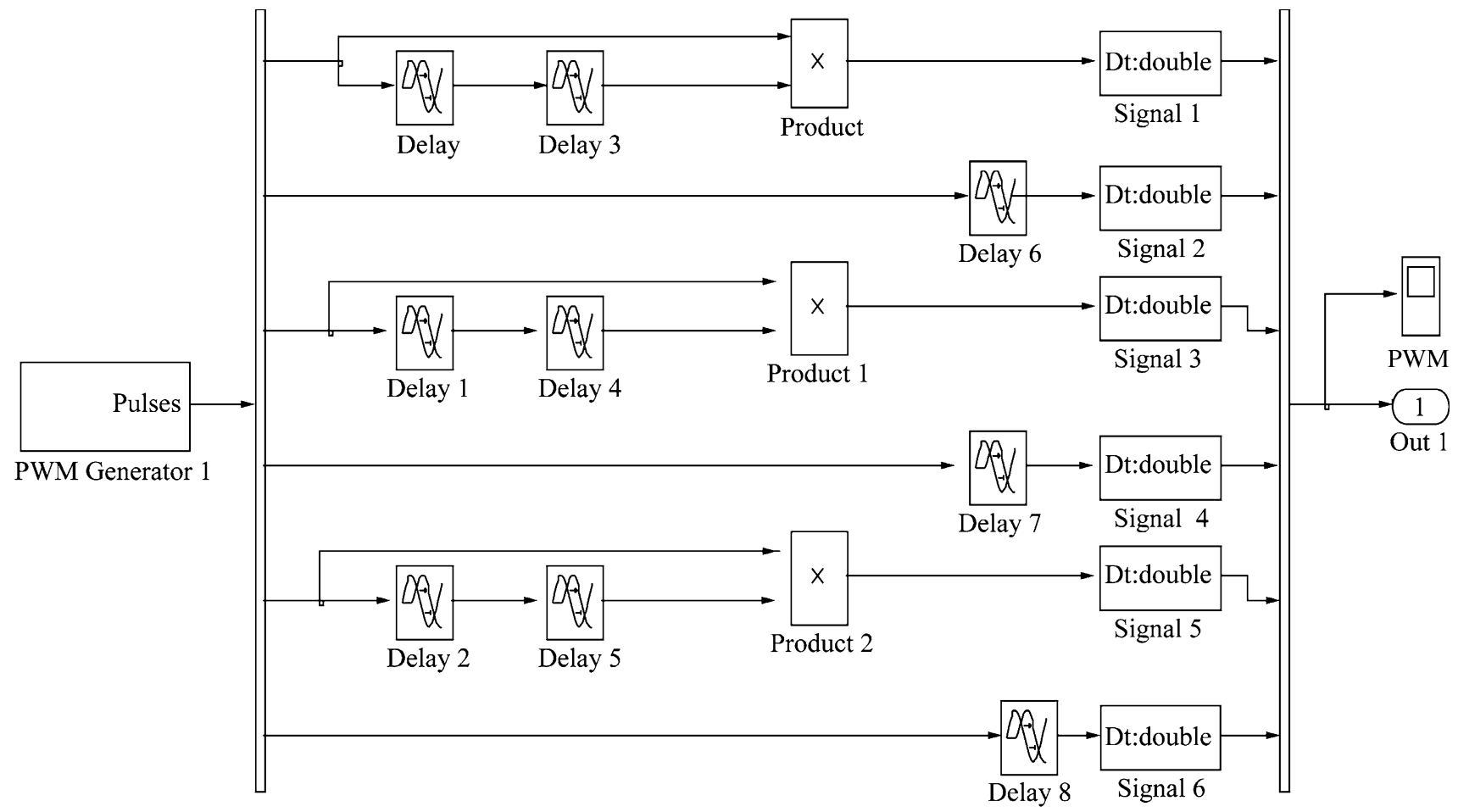

Fig. 7 The simulation model of SPWM with dead zone 


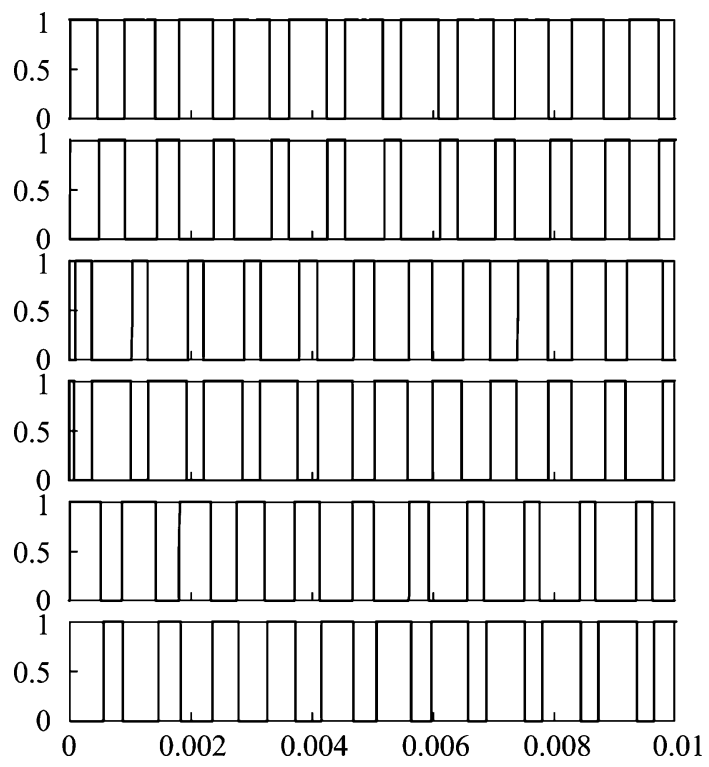

Fig. 8 The PWM waveform at the dead zone time of $10 \mu \mathrm{s}$

Figure 7 is the SPWM simulation model with the dead zone, and the dead time is set in the delay module. Figure 8 is the SPWM waveform of six bridge arms when the dead time is set at $10 \mathrm{~ms}$.

The speed map of mine hoist is divided into five stalls, corresponding five different frequency values are set by $4,10,20,41$ and $50 \mathrm{~Hz}$. The inhibition effect of harmonic circulating current by instantaneous voltage feedback method is verified in five frequencies. The difference of dead time of the two-conversion units is set by $3 \mu \mathrm{s}$, and the simulation results controlled by instantaneous outputting voltage feedback are shown in Fig. 9.

The Fig. 10 shows the outputting voltage harmonic distortion rate by open-loop.

By comparing the results from Figs. 9 and 10, the using of the instantaneous outputting voltage feedback method effectively inhibits the harmonic circulating current at different frequencies. When the outputting voltage is $4 \mathrm{~Hz}$, the harmonic distortion rate of the outputting voltage is $5.05 \%$ under the open-loop state, but the harmonic distortion rate is $2.32 \%$ of the controlled by the instantaneous outputting voltage feedback. When the frequency increases, the harmonic distortion decreases. The harmonic distortion rate reaches the minimum value at frequency $50 \mathrm{~Hz}$.

Fig. 9 The harmonic distortion rate controlled by the instantaneous outputting voltage feedback
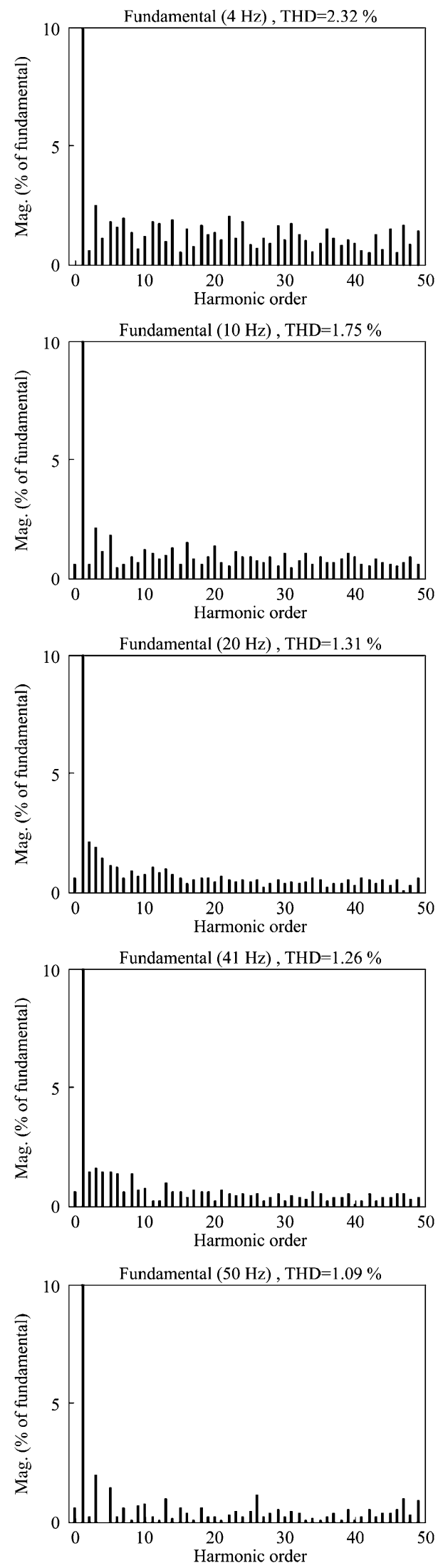

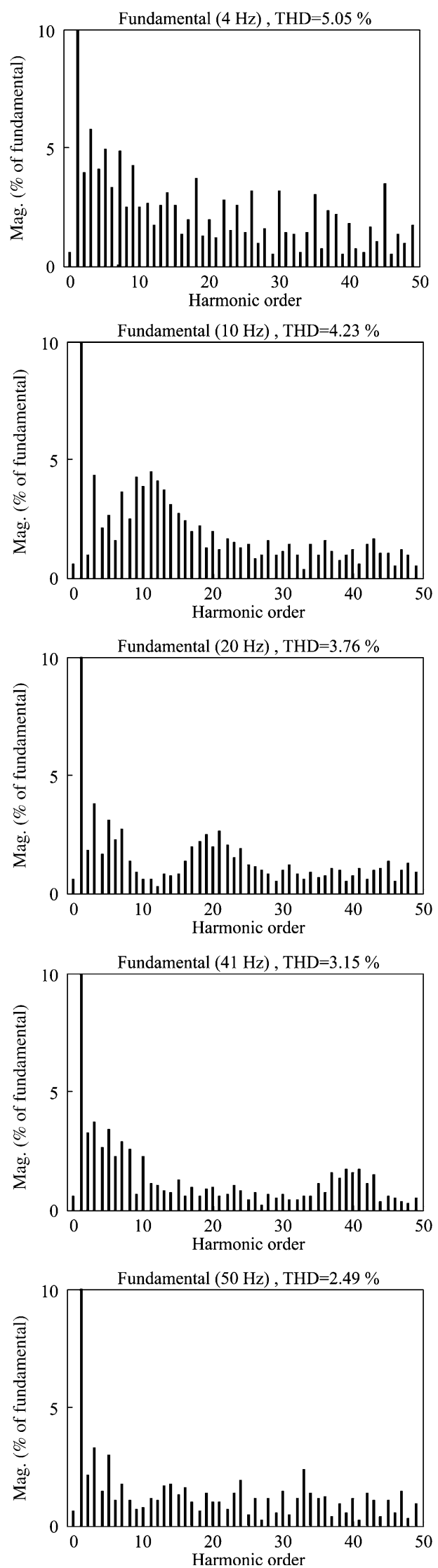

4Fig. 10 The harmonic distortion rate by open-loop

\section{Conclusions}

(1) By establishing the transfer function of the parallel inverters controlled by the close-loop adjustment of the instantaneous voltage feedback, the influence of the parameters of the close-loop feedback circuit to the inhibition effects to the outputting circulation current are observed. As the increasing of the parallel inductance, the filter inductor and the equivalent circuit resistance, the inhibition effect of the outputting circulation current are more obvious. However, the drop and the outputting waveform distortion of the voltage should be greater when the parameters of the parallel inductance, the filter inductor and the equivalent circuit resistance are selected as high enough. Furthermore, the inhibition effect of the outputting circulation current is mainly adjusted by the regulator of the instantaneous voltage feedback.

(2) Through introducing the proportion integration (PI) controller into the close-loop adjustment by the instantaneous voltage feedback, the characteristic equation is gained to determine the PI parameters. The Bode plots have been drawn to investigate the influence of the dominant roots of the characteristic equation to the inhibition effects of the controller, which determine the PI parameters by the selected values of the damping ratio, natural frequency and the normal number.

In the established simulation model of parallel inverter system, the harmonic distortion rate at the outputting voltage frequency values of $4,10,20,41$ and $50 \mathrm{~Hz}$ are calculated to verify inhibition effects of the outputting circulation current by the closeloop controller of the instantaneous voltage feedback. The harmonic distortion rate are all low than $2.32 \%$ of the controlled by the instantaneous outputting voltage feedback.

Open Access This article is distributed under the terms of the Creative Commons Attribution 4.0 International License (http://creati vecommons.org/licenses/by/4.0/), which permits unrestricted use, distribution, and reproduction in any medium, provided you give appropriate credit to the original author(s) and the source, provide a link to the Creative Commons license, and indicate if changes were made.

\section{References}

Chen LL, Xiao L, Hu WB, Yan YG (2003) Reasons and restraining methods for DC bias in inverter output voltage. Power Electron 37:27-29 
Chen LL, Xiao L, Gong CY, Yan YG (2004) Reasons and methods for detecting and restraining DC circulating current in parallel inverter system. Proc CSEE 24:56-61

He GF, Xu DH (2012) The DC circulation control strategy based on decoupled control scheme in the multi-module paralleled inverter system. Proc CSEE 32:43-51

He CH, Zhang XY (2008) Analysis and solution of DSP controls for the single-phase UPS parallel DC loop-current. Telecom Power Technol 25:48-50

Hyosung K, Ji JK (2004) Novel topology of a line interactive UPS using PQR instantaneous power theory. Ind Appl Conf 4:2232-2235

Kawakami N, Honbu M, Ikimi T (1994) Quick response and lowdistortion current control for multiple inverter-fed induction motor drives. IEEE Tans Power Electron 9:240-247
Liu YB, Ding ZH, Yao YF, Cheng SH (2011) Research of harmonic circulating current restrain in parallel inverter. Chin J Power Sources 35(1):79-81

Ogasawara S, Takagaki J, Akagi H (1992) A novel control scheme of a parallel current-controlled PWM inverter. IEEE Trans Ind Appl 28:1023-1030

Zhang Y, Duan SX, Kang Y, Chen J (2006a) The research of harmonic circulating current restrain in parallel inverter systems. Proc CSEE 26:67-72

Zhang Y, Duan SX, Kang Y, Chen J (2006b) The research of zerosequence circulating currents between parallel three-phase inverters. Proc CSEE 26:62-67 